\title{
Diseño de un convertidor de energía undimotríz en baja escala
}

\author{
Design of a small-scale wave power converter
}

\author{
Janeth Alcalá $^{a}$, Jorge Gudiño-Lau $^{b}$, Saida Charre ${ }^{c}$, Miguel Durán $^{d}$, Daniel Vélez. ${ }^{e}$
}

\begin{abstract}
:
In this work, the proposal for the design of an experimental prototype that allows generating electricity through the use of wave energy is presented. Throughout the work the conditions of interest for the waves are addressed, the current systems that have been developed for capturing wave energy, the design of the proposal is presented and the experimental results are evaluated with a small-scale prototype.
\end{abstract}

\section{Keywords:}

Energy generation, marine energy, wave energy

Resumen:

En este trabajo se presenta la propuesta del diseño de un prototipo experimental que permite generar electricidad mediante el aprovechamiento de la energía undimotríz. A lo largo del trabajo se abordan las condiciones de interés para el sistema oleaje, los sistemas actuales que se han desarrollado para captación de energía undimotríz, se presenta el diseño de la propuesta y los resultados experimentales se evalúan con un prototipo a baja escala.

\section{Palabras Clave:}

Generación de energía, energía marina, energía undimotríz

\section{Introducción}

La energía eléctrica forma parte del desarrollo de una sociedad y uno de sus parámetros de interés más estudiados es la demanda de energía eléctrica. De acuerdo a la gráfica de demanda que se actualiza en tiempo real y se revisa por el Centro Nacional de Control de Energía en México (CENACE) [1], a la fecha, la demanda bruta actual para el Sistema Interconectado Nacional es de 37, 452 MW. Datos publicados al 2018 reflejan que el $73.5 \%$ de la energía eléctrica que se genera en el mundo proviene de energías convencionales, tales como los combustibles fósiles y la energía nuclear. El resto, es decir el $26.5 \%$, se obtiene a partir de fuentes no convencionales, entre ellas las provenientes de recursos renovables, entre las cuales se pueden mencionar la energía hidráulica, geotérmica, solar, eólica, biomasa y oceánica o marina [2]. No obstante, con el objetivo de reducir la generación de las emisiones de gases de efecto invernadero (GEI) al calentamiento global y cumplir con lo proyectado en el Acuerdo de París, se han hecho esfuerzos conjuntos en tre países por reducir la generación de energía a partir de fuentes convencionales y se busca aumentar la producción a partir de fuentes no convencionales. En este aspecto, México se une a estas iniciativas y busca satisfacer las necesidades energéticas del país y disminuir las emisiones de contaminantes. De acuerdo a los datos publicados en el Programa de

\footnotetext{
a Autor de Correspondencia, Universidad de Colima, http://orcid.org/0000-0002-0238-3952, Email: janethalcala@ucol.mx

b Universidad de Colima, https://orcid.org/0000-0002-0585-908X, Email: jglau@ucol.mx

c Universidad de Colima, https://orcid.org/0000-0002-3823-5388, Email: scharre@ucol.mx

d Universidad de Colima, https://orcid.org/ 0000-0002-0780-6192, Email: mduran@ucol.mx

e Universidad Autónoma del Estado de Hidalgo, https://orcid.org/0000-0001-6488-2960, Email: daniel@uaeh.edu.mx
} 
Desarrollo del Sistema Eléctrico Nacional (PRODESEN) durante 2018 en México la energía producida por tecnología fue de 317, 278 GWh con la distribución mostrada en la Figura 1, de donde se observa que el 23.2 $\%$ de la producción de energía se generó a partir de fuentes no convencionales [3]. Se puede además identificar en la Figura 1, la au sencia de producción a partir de fuentes oceánicas o marinas, aun y cuando México es un país privilegiado cuyo mapa muestra una superficie oceánica del $65 \%$ mayor a la terrestre que en comparación ocupa el 35\% [4], con costas que se extienden a lo largo de $11,122 \mathrm{~km}$ sin considerar el territorio in sular; limitando con el Océano Pacifico, el Golfo de California, el Golfo de México y el Mar Caribe, evidenciando por lo tanto un recurso energético potencialmente explotable.

La energía oceánica se obtiene a partir de la energía potencial, cinética, térmica o química del agua de mar [56] generando cinco formas de aprovechamiento que se clasifican como energía: mareomotriz o energía de las mareas, undimotríz (u ola motriz), osmótica, térmica oceánica y de las corrientes marinas. La energía a partir de fuentes oceánicas es una fuente inagotable e intermitente de energía, 5 veces más concentrada que la energía eólica y 10-30 veces más alta que la energía solar.

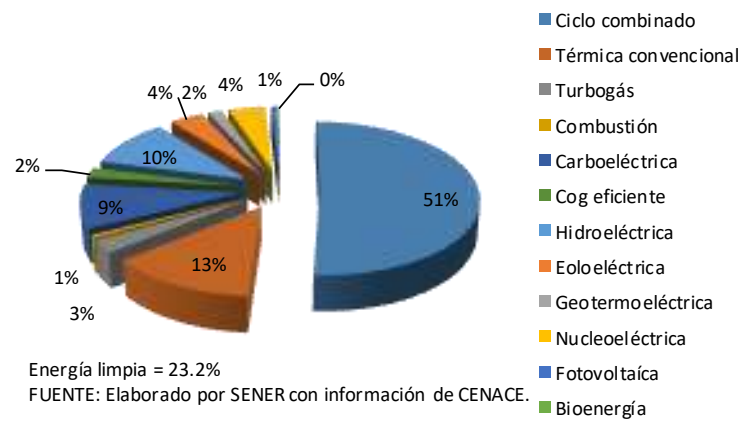

Figura 1. Energía producida en 2018 por tecnología [3].

En 2017 inició actividades el Centro Mexicano de Innovación en Energía del Océano (CEMIE-Océano) con la finalidad de fomentare impulsar la investigación y el desarrollo tecnológico en el país y dirigido a la extracción, conversión y distribución de las diversas formas de la energía marina [7]. A través de los estudios realizados por el CEMIE-Océano, se han realizado diversos estudios para evaluar el potencial que se tienen en México y se han desarrollado algunos prototipos de prueba en conjunto con las instituciones participantes y se prevé que en corto plazo se comiencen a desarrollar los proyectos de generación. Además, a través de estudios realizados, se ha identificado que en cuanto a las clasificaciones de generación de energías oceánicas en México ofrecen un mayor potencial la energía mareomotriz y la undimotríz [6]. En la actualidad México no cuenta con desarrollos comerciales para la generación de electricidad a partir de fuentes oceánicas. Sin embargo en 2012 se licitó un proyecto piloto para generar energía undimotríz con capacidad de $3 \mathrm{MW}$ en las costas de Rosarito, Baja California ubicándose frente a las cosas de la Central Termoeléctrica Presidente Juárez, a 20 kilómetros al suroeste de Tijuana [8]. Además, en 2017 la compañía israelí Eco Wave Power con sede en Tel Aviv anunció la construcción de una planta de energía undimotríz de 4.1 MW aprovechando la energía mecánica generada por el movimiento de las olas en las costas de Manzanillo en el estado de Colima [9]. Sin bien actualmente ambos proyectos se encuentran detenidos, es claro que en México existe un interés genuino por incluir dentro de sus medios de generación las energías oceánicas involucrando tanto al sector gubernamental, como al industrial y académico. Por ello, en este trabajo se presenta la propuesta del diseño de un prototipo experimental que permite generar electricidad mediante el aprovechamiento de la energía undimotríz. A lo largo del trabajo se abordan las condiciones de interés para el oleaje, los sistemas actuales que se han desarrollado para captación de energía undimotríz, se presenta el diseño de la propuesta y los resultados experimentales se evalúan con un prototipo a baja escala.

\section{El poder del oleaje}

Las olas son consideras como movimientos ondulatorios u oscilaciones periódicas de la superficie del mar, formadas por crestas y depresiones que se desplazan horizontalmente y que pueden ser descritas en función a: su periodo $(T)$, longitud de onda $(\lambda)$, altura $(H)$ pendiente (s), número de onda $(k)$, frecuencia angular $(\omega)$ y la profundidad de lo que se denomina la columna de agua (h). Además, se establece una relación entre la profundidad y la longitud de onda y que se utiliza para determinar el comportamiento de las partículas del fluido y de la forma de la onda, estableciendo tres clasificaciones como se muestra en la Figura 2 y se describe a continuación [10]:

- Aguas poco profundas o someras: las partículas de fluido describen órbitas que se consideran casi planas y la profundidad del agua es menor a 1/20 de la longitud de onda $\mathrm{h}<\lambda / 2$.

- Aguas transición: en estas, las partículas del fluido describen órbitas elípticas y la profundidad del agua se ubica en un intervalo $\lambda<h<\lambda / 2$.

- Aguas profundas:las partículas describen órbitas circulares y el oleaje se propaga sin interacción con el fondo, $h>\lambda / 2$. 
Dada la propagación del oleaje desde aguas profundas hasta las someras, el oleaje tiene a disminuir su velocidad de propagación, disipando además la energía como causa de la fricción que se genera con el fondo marino, por lo que se considera que las aguas profundas son más atractivas para la producción de energía, siempre y cuando el método de captación sea el adecuado para conservar el potencial de generación.

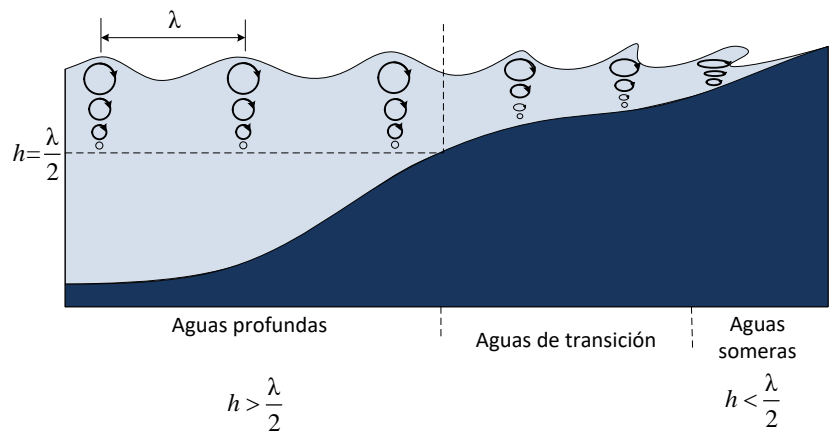

Figura 2. Clasificación de la programación de las olas, adaptado de [10].

Por otro lado, en la Figura 3 se muestra las formas de onda características para una ola, en donde es posible identificar la altura y el periodo de la ola.

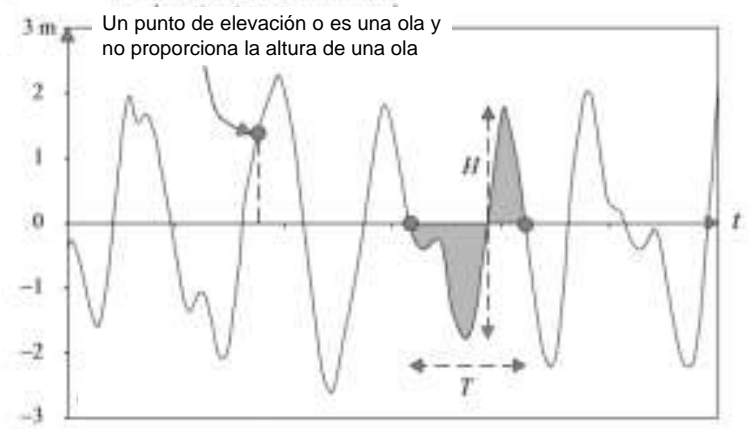

Figura 3. Clasificación de convertidores de energía del oleaje, adaptado de [11].

\section{Sistemas de captación de energía de las olas}

En referencia a los métodos de extracción de energía de las olas, a la fecha se han propuesto diferentes prototipos para convertidores de energía del oleaje (CEO) que se pueden clasificar en gran medida en cuanto al principio de trabajo, número de grados de libertad, tipo de anclaje, tamaño y ubicación u orientación en relación a la dirección de propagación del oleaje, como se muestra en la Figura 4. Entre los de componentes de un CEO se identifican: 1) el sistema de captación de energía, 2) el de extracción de energía, 3) el sistema de fuerza y 4) el elemento de conversión de energía eléctrica [10].
En este trabajo se aborda el funcionamiento del elemento oscilante con dispositivos de traslación vertical y que se pueden colocar sobre o por debajo del nivel medio del mar para extraer la energía del oleaje partiendo del movimiento de traslación vertical de un elemento flotador que es provocado por la oscilación de la superficie del mar. Este tipo de dispositivos se suele ubicar en aguas profundas y se clasifica como offshore (lejos de la costa) flotante, grupo 5, como se muestra en la Figura 4.

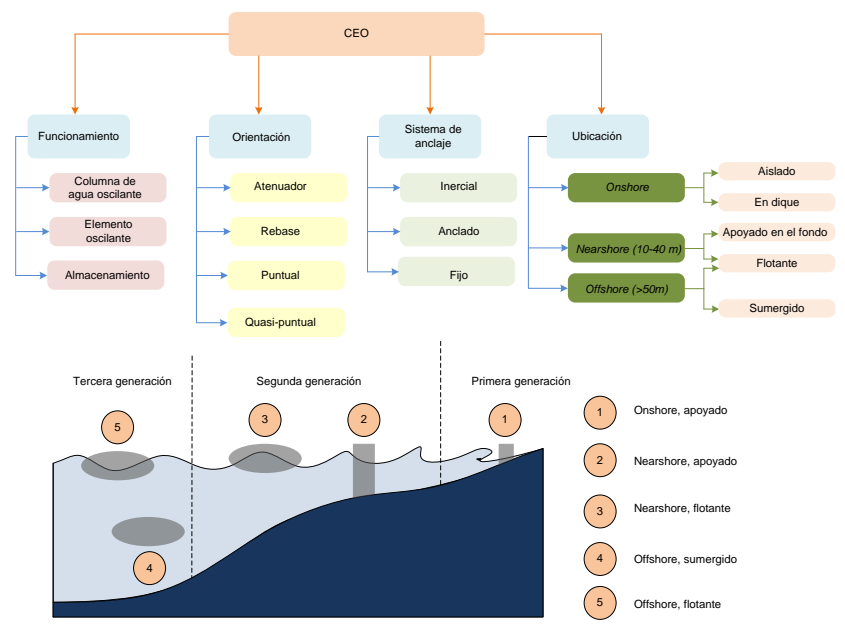

Figura 4. Clasificación de convertidores de energía del oleaje, adaptado de [10].

\section{Propuesta CEO}

En la Figura 5 se muestra el diagrama general que ejemplifica la propuesta del trabajo y que se compone de los cuatro bloques identificados en un CEO. El sistema de captación de energía se compone por un canal de oleaje y un sistema que se utiliza para generar del oleaje. El bloque del canal de oleaje será el encargado de producir el movimiento ondulatorio de las olas para producirque se captara utilizando un sistema de boyas y que se encargarán de transmitir el movimiento mecánico a un bloque de fuerza para posteriormente tradu cirlo a sistema de generación eléctrica.

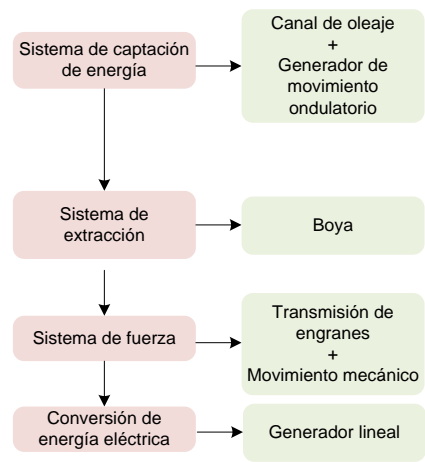


Figura 5. Diagrama a bloques para el sistema CEO propuesto.

En las figura 6a y $6 \mathrm{~b}$ se muestra una vistas laterales del sistema propuesto y diseñado para el CEO con el software SolidWorks ${ }^{\circledR}$. Las medidas del sistema son $140 \mathrm{~cm}$ de largo por $70 \mathrm{~cm}$ de alto y $50 \mathrm{~cm}$ de profundidad, con una capacidad de almacenamiento de 490 litros y para la estructura se propone utilizar vidrio templado. Para generar el movimiento ondulatorio se utiliza una base vertical que funciona como un mecanismo lineal empujado por un motor de corriente directa que se utiliza para provocar el movimiento ondulatorio y que simula el movimiento de un pistón empujando el mecanismo por medio de unos eslabones que se soldaron al motor.

Para el sistema de extracción se utiliza el principio de una boya electrógena, en el que la boya oscila continuamente, subiendo y bajando; al igual que lo hacen las olas. Cada molécula de la masa liquida oscilante perpendicularmente vuelve cuando pasa la ola al mismo sitio donde se encontraba, generando un vaivén con una componente vertical, de arriba a abajo, y otra longitudinal. La boya integra un núcleo magnético con forma de pistón y que forma la médula de la columna vertebral de la boya vinculando el movimiento de la boya con el ritmo oscilatorio de las olas y permitien do la generación de una corriente y fijando una tensión a través del campo magnético generado. En el sistema de fuerza se incluye un mecanismo de engranajes para transmitir fuerza al movimiento obtenido de la boya.

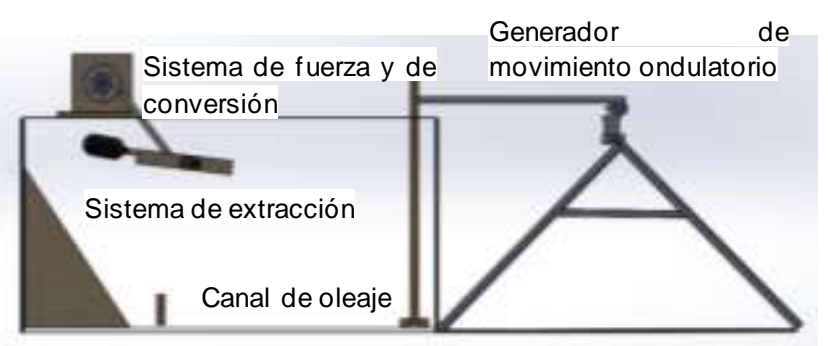

(a)

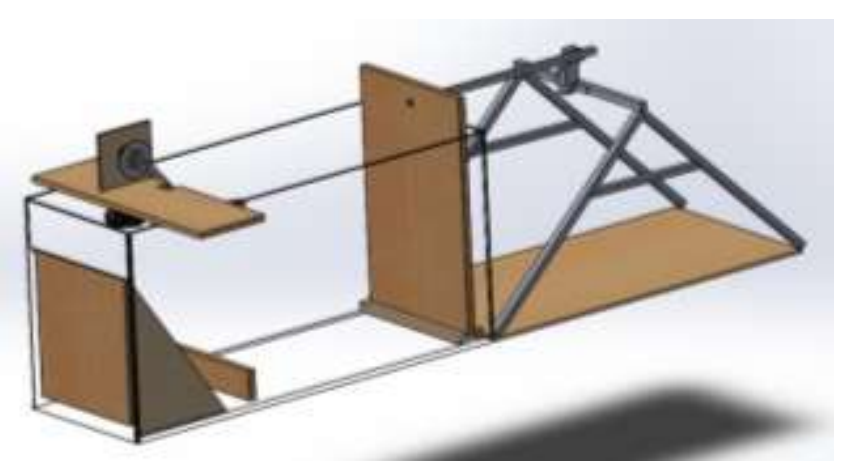

(b)

\section{Figura 6. Diagrama a bloques para el sistema CEO propuesto.}

Para el sistema de fuerza y conversión se utilizó el principio de operación de una manivela biela corredera con la finalidad de transformar el movimiento circular en un movimiento de traslación. El mecanismo se crea con dos barras unidas por una unión de revoluta, en donde el extremo encargado de rotar la barra (la manivela) se une a un punto fijo en el centro de giro y el otro extremo se une a la biela. El extremo restante de la biela se une a un pistón que se mueve en línea recta. En la Figura 7 se muestra el diseño propuesto para la manivela, formada por una estructura rectangular unida a un disco en el que se colocarán 24 imanes de neodimio N52 y que servirá para generar la energía, las medidas están dadas en cm.

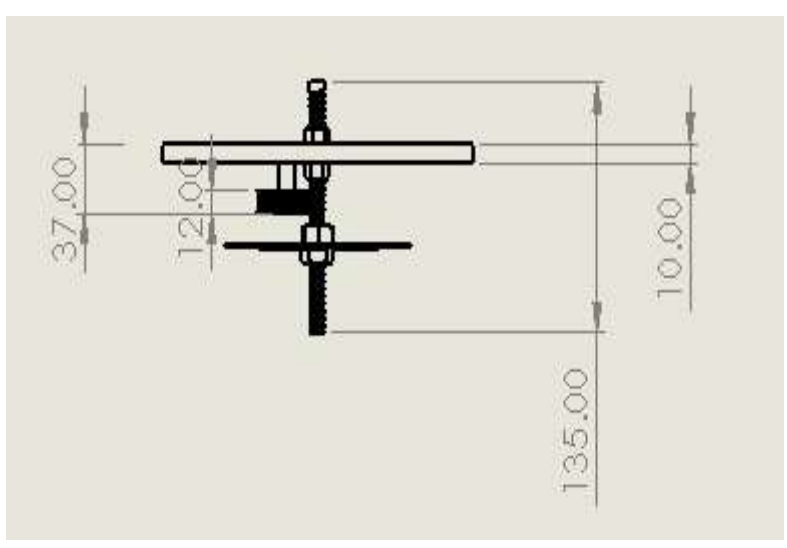

(a)

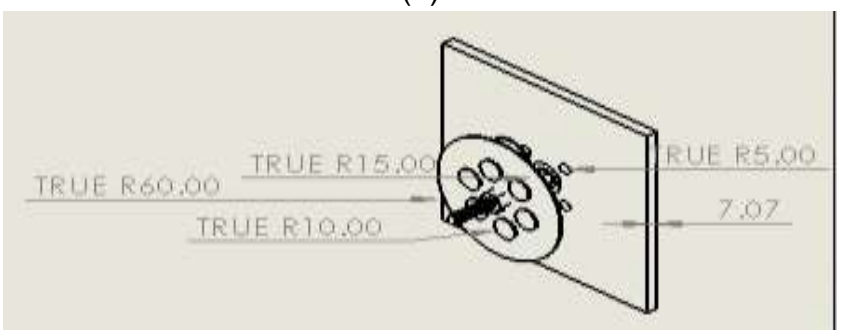

(b)

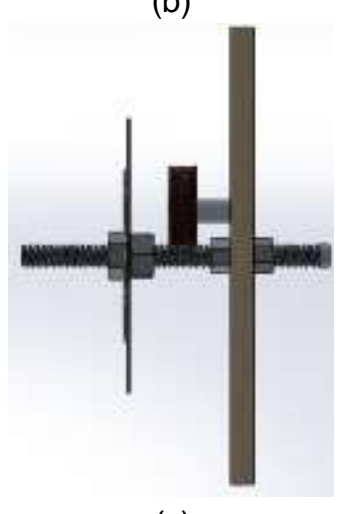

(c)

Figura 7.Diseño para la manivela integrada al CEO. 
El mecanismo de engranajes, está formado por dos ruedas dentadas, formando un par de contacto lineal, unidas mediante un cierre de cadena y para las ruedas dentadas engranadas, se tiene la siguiente ecuación que relaciona las velocidades angulares con el radio:

$$
I=\frac{\omega_{2}}{\omega_{1}}=\frac{R_{2}}{R_{1}}
$$

donde:

I = Relación de transmisión

$\omega_{1}=$ Velocidad angular de la primera rueda

$\mathrm{R}_{1}=$ Radio primitivo de la primera rueda

$\omega_{2}=$ Velocidad angular de la segunda rueda

$\mathrm{R}_{2}=$ Radio primitivo de la segunda rueda

La ecuación anterior se puede expresar también en función del número de dientes $(z)$ de la siguiente manera:

$$
I=\frac{\mathrm{z}_{1}}{\mathrm{z}_{2}}=\frac{\omega_{2}}{\omega_{1}}
$$

donde:

I = Relación de transmisión

$Z_{1}=$ Número de dientes de la primera rueda

$Z_{2}=$ Número de dientes de la segunda rueda

Por tanto, la relación de transmisión es igual a la inversa de la razón de dientes. La caja de engranes transmite el movimiento circular a un sistema de inductores que se conectan de tal manera que cuando gira el disco de imanes se transmiten los cambios. En la Figura 8 se muestra el sistema de engranes acoplado a la base del disco de imanes y en la Figura 9 se muestra la conexión de la base del disco de imanes con la boya.

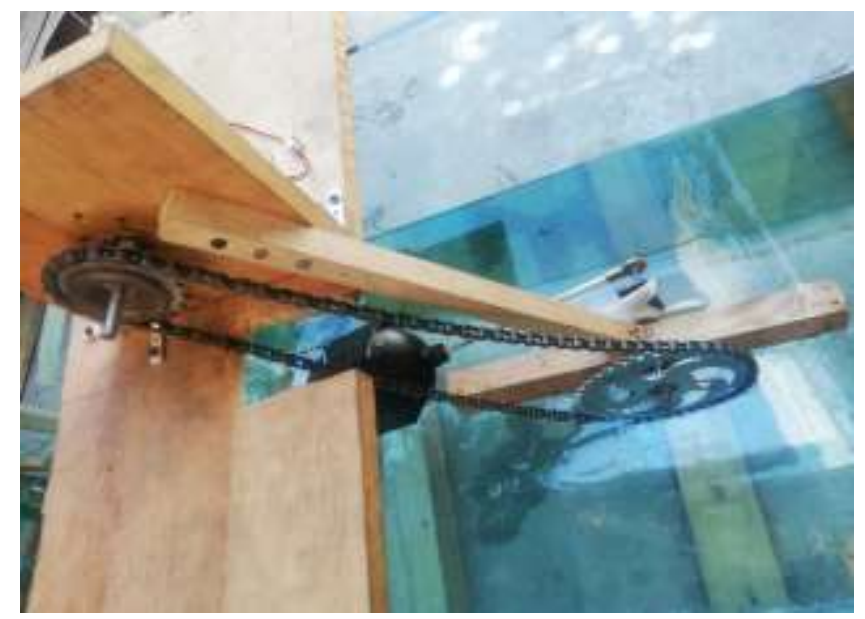

Figura 8.Engrane y cadena ensamblado en la base del disco de imanes.

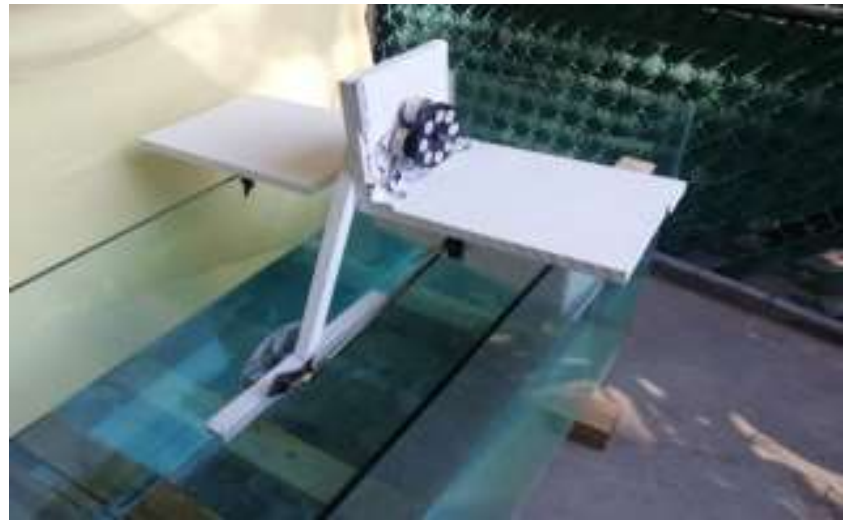

Figura 9.Conexión de disco de imanes con el mecanismo de laboya.

En la Figura 10 se muestra la versión preliminar para el prototipo CEO propuesto, en el sistema se pueden observar la integración de las diferentes secciones.

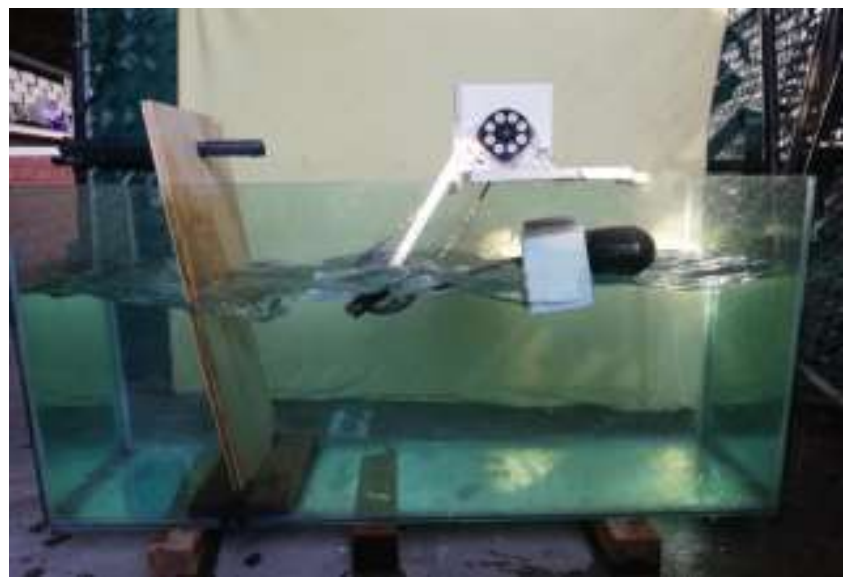

Figura 10.Versión preliminar para el prototipo CEO.

\section{Resultados experimentales}

Con el prototipo CEO propuesto es posible generar olas con una altura máxima de $15 \mathrm{~cm}$ considerando una altura de llenado de $50 \mathrm{~cm}$ y tomando una altura mínima de 10 $\mathrm{cm}$. Para estos datos, a continuación se calcula la potencia energética. No obstante, es importante aclarar para la estimación del potencial de energía de las olas se debe tomar en cuenta que el principio del oleaje es complejo y que considera numerosos trenes de olas que son no regulares con distintos valores de periodo, altura y dirección. Si se considera una forma sinusoidal defina por un período (1/f), entonces es posible calcular la energía de las olas que se transportan con una velocidad de energía igual a la velocidad de un grupo de olas $C_{g}(g T / 4 \pi)[11]$ y 
para una ola de mar, el nivel de potencia se puede expresar en términos del espectro de ondas como:

$$
P_{w}=\rho g \int_{0}^{\infty} C_{g}(f) S(f) d f=\frac{\rho g^{2} T_{e} H_{s}^{2}}{64 \pi}
$$

donde:

$\rho$ es la densidad de la masa del agua del mar con un valor de $1.025 \mathrm{~kg} / \mathrm{m}^{3} ; \mathrm{g}$ es la aceleración de la gravedad con un valor de $9.81 \mathrm{~m} / \mathrm{s}^{2} ; \mathrm{T}_{e}$ es el periodo de energía; $\mathrm{H}_{\mathrm{s}}$ es la altura significante de la ola; $\mathrm{C}_{g}(\mathrm{f})$ es la celeridad de grupo y que indica la velocidad del grupo de olas y $s(f)$ es el espectro de olas sin tener en cuenta la dirección, describiendo cuantitativamente cómo las diferentes frecuencias de onda f contribuyen a la energía. Tal que si se integran estos datos al prototipo CEO, considerando el peor de los casos, con una altura de ola mínima de de 10 $\mathrm{cm}$, se tiene que $\mathrm{T}_{e}=1 \mathrm{~s}$ y $\mathrm{H}_{\mathrm{s}}=10 \mathrm{~cm}(0.1 \mathrm{~m})$. Por lo que:

$$
P_{w}=4.90 \mathrm{~W} / \mathrm{m}
$$

En la Figura 11 se muestra el resultado obtenido al medir con un osciloscopio la tensión generada para un oleaje bajo, con olas de altura de $10 \mathrm{~cm}$ para este caso, se obtuvo una tensión de salida de $7.20 \mathrm{~V}$, con una frecuencia de es $3.63 \mathrm{~Hz}$. De forma similar, en la Figura 12 se muestran los resultados para un escenario en el que se contemplan condiciones de oleaje medio, con olas de altura de $13 \mathrm{~cm}$, para esta condición se obtuvo un periodo promedio de alrededor de 180 ms con una tensión de salida promedio para las pruebas de $17.8 \mathrm{~V}$. Por último, en la figura 13 se muestran los resultados obtenidos cuando se realizan pruebas generando condiciones de oleaje con una altura de $15 \mathrm{~cm}$, la Figura 13 es una figura representativa de los resultados obtenidos, obteniéndose tensiones de salida de 40V con señales periódicas de 90 ms. Los resultados obtenidos se resumen en la Tabla 1.

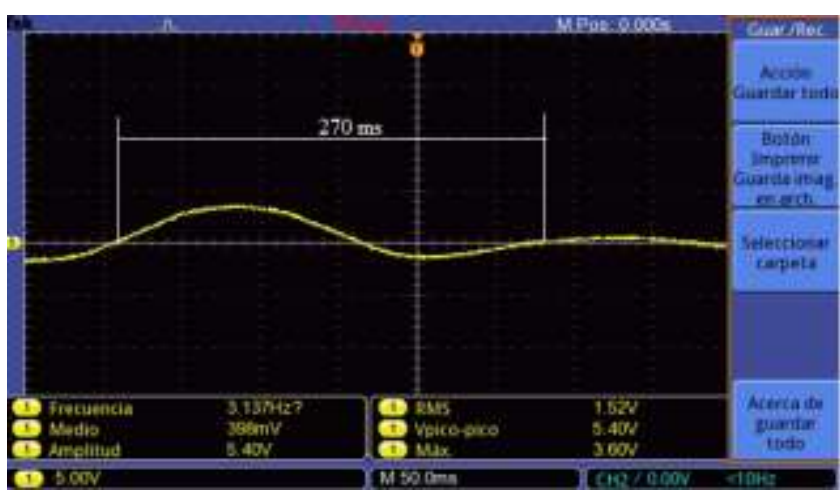

Figura 11.Resultados obtenidos con el sistema CEO cuando se evalúan condiciones de bajo oleaje.

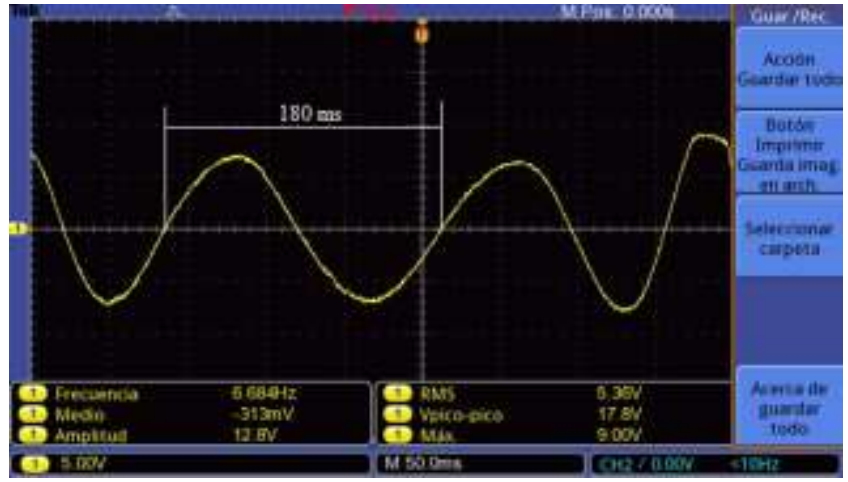

Figura 12.Resultados obtenidos con el sistema CEO cuando se evalúan condiciones de oleaje medio.

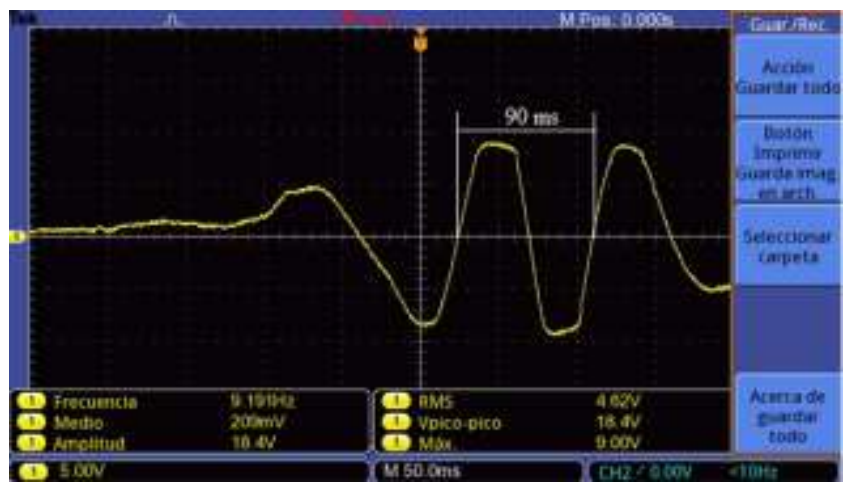

Figura 13.Resultados obtenidos con el sistema CEO cuando se evalúan condiciones de oleaje alto.

\begin{tabular}{|c|c|c|c|}
\hline \multicolumn{4}{|c|}{ Escenario de oleaje bajo } \\
\hline \multicolumn{2}{|c|}{ Parámetros de salida del CEO } & \multicolumn{2}{|c|}{ Condiciones de oleaje } \\
\hline $\mathrm{V}$ & $7.20 \mathrm{~V}$ & Altura $(\mathrm{H})$ & $10 \mathrm{~cm}$ \\
\hline$f$ & $3.7 \mathrm{~Hz}$ & Pruebas & $1.595 \mathrm{~s}$ \\
\hline $\mathrm{Te}$ & $270 \mathrm{~ms}$ & & \\
\hline \multicolumn{4}{|c|}{ Escenario de oleaje medio } \\
\hline \multicolumn{2}{|c|}{ Parámetros de salida del CEO } & \multicolumn{2}{|c|}{ Condiciones de oleaje } \\
\hline V & $17.8 \mathrm{~V}$ & Altura $(\mathrm{H})$ & $13 \mathrm{~cm}$ \\
\hline$f$ & $5.55 \mathrm{~Hz}$ & Pruebas & $1.693 \mathrm{~s}$ \\
\hline$\overline{T e}$ & $180 \mathrm{~ms}$ & & \\
\hline \multicolumn{4}{|c|}{ Escenario de oleaje alto } \\
\hline \multicolumn{2}{|c|}{ Parámetros de salida del CEO } & \multicolumn{2}{|c|}{ Condiciones de oleaje } \\
\hline $\mathrm{V}$ & $40 \mathrm{~V}$ & Altura $(\mathrm{H})$ & $15 \mathrm{~cm}$ \\
\hline$f$ & $11.11 \mathrm{~Hz}$ & Pruebas & $1.768 \mathrm{~s}$ \\
\hline$\overline{\mathrm{Te}}$ & $90 \mathrm{~ms}$ & & \\
\hline
\end{tabular}

Tabla 1. Resumen de resultados obtenidos con el CEO. 


\section{Conclusiones}

La energía denominada undimotríz, es una de las formas de obtener energía renovable más prometedora de las energías oceánicas. En la actualidad existen varios dispositivos que extraen energía de las olas, siendo la tecnología de columnas oscilantes de agua una de las que más se adapta a las características de la costa de Colima. Por ello en este trabajo se planteó como propuesta diseñar un prototipo experimental que permitiera generar electricidad mediante el aprovechamiento de la energía cinética de un canal de oleaje a baja escala. A partir de los resultados obtenidos, fue posible producir un potencial energético mínimo de $4.90 \mathrm{~W} / \mathrm{m}$ considerando alturas mínimas de $10 \mathrm{~cm}$. Los resultados obtenidos son preliminares pues el prototipo aún se encuentra en la etapa de prueba, como trabajo a futuro se espera incluir una etapa que permita interconectar un sistema en baja escala a la red eléctrica.

\section{Referencias}

[1] "Grá fica de Demanda", Cenace.gob.mx, 2020. [En línea]. Disponible en: https://www.cenace.gob.mx/GraficaDemanda.aspx. [Accesado: 310- 2020].

[2] Silva, R., Posada, G., Félix, A., "Boletín de difusión de las actividades del Centro Mexicano de Innovación en Energía Océano", Centro Mexicano de Innovación en Energía Océano, Año 1, No. 2, pp. 1-38, 2017.

[3] SENER, "Programa de Desa rrollo del Sistema Eléctrico Nacional20182033”, gob.mx, 2020. [En línea]. Disponible en: https://www.gob.mx/sener/articulos/prodesen-2019-2033-221654. [Accesado: 7-10-2020].

[4] González Huerta, RG., J.M. SandovalPineda, J. N. Hernández Pérez, E. Álvarez del Rio, 2017. Interconexión a la Red y Sistemas de Almacenamiento de Energía. Estado del Arte. Cemie-Océano, Universidad Autónoma de Campeche.

[5] González-Ramírez, X., Hernández-Robles, I. A., \& Barrios- Piña, H. (noviembre-diciembre, 2017). Potencial energético undimotríz en nodos costeros de México. Parte 1: estimación energética. Tecnología y Ciencias del Agua, 8(6), 5-22.

[6] Gómez, M., León, E., Palacios, L. M., Campos, R., “Alternativas para el aprovechamiento de energías renovables en México: energía undimotríz", in XXV Congreso Internacional Anual de la SOMIM, Mazatlán, Sinaloa, México. Septiembre 2019, pp. 1-8.

[7]CEMIE-Océano, [En línea]. Disponible en b: https://cemieoceano.mx/index.html . [Accesado: 5-10-2020]

[8] “Marersa construirá la centralde energía undimotríz en Rosario”, 2012. [En línea]. Disponible en: http://monitoreconomico.org/noticias/2012/aug/07/marersa -construirala-central-de-energia-undimotriz-en-rosarito/. [Accesado: 05-10-2020]

[9] "La firma Eco Wave Power construirá planta eléctrica en México", 2017. [En línea]. Disponible en: https://es.israel21c.org/la-firma-eco- wa ve-power-construira-planta-electrica-en-mexico/. [Accesado: 05-102020].

[10] Galván, E., “Análisis de la dinámica delmovimiento de un convertidor de energía del oleaje con más de un grado de libertad", tesis doctoral, CICESE, 2019[En línea]. Disponible en: http://cicese.repositorioinstitucional.mx/jspui/handle/1007/2820.[Acce dido: 15-10-2020].

[11] X. González-Ramírez, H. Barrios-Piña and I. A. Hernandez-Robles, "Wave energy conversion assessment at Mexican seaside points," 2016 IEEE PES Transmission \& Distribution Conference and ExpositionLatin America (PES T\&D-LA), Morelia, 2016, pp. 1-6, doi: 10.1109/TDC-LA.2016.7805625. 